\title{
Semi-Automated Micro Assembly for Rapid Prototyping of a One DOF Surgical Wrist*
}

\author{
Ranjana Sahai, Jusuk Lee, and Ronald S. Fearing \\ $\{$ rsahai, ronf\}@eecs.berkeley.edu \\ Department of EECS, University of California, Berkeley, CA 94720. USA
}

\begin{abstract}
We have developed new methods for the automated assembly of prototype structures and we illustrate them with the construction of a simple one DOF $5 \mathrm{~mm}$ surgical wrist employing polyester flexures instead of revolute joints. The first step in the structural assembly involves the construction of hollow stainless steel triangular beams that are used for the rigid elements of the structure. It includes the development of a folding fixture to bend stainless steel sheets and the determination of a folding angle sequence by static analysis using a compliant mechanism model. The semi-automatic process of using a millirobot (Orthotweezers) to manipulate and assemble the beams and attach the flexures is described in detail. The paper ends with a description of the procedure used to design the wrist.
\end{abstract}

\section{Introduction}

This paper describes a semi-automated procedure for rapid prototyping of millimeter-scale robotic structures and demonstrates it by building a prototype for a one DOF surgical wrist. The long-term goal of this work is to fully automate the fabrication of such structures. This envisioned automated assembly would include the construction of the structure (the body of the robot) and the attachment of actuators, sensors, and any necessary wiring. Because the techniques in the assembly of structures these sizes are largely undeveloped, this goal encompasses a considerable amount of uncharted territory. Thus, as a first step, the current work focuses on automating the construction of the 3-D structure. More specifically, it focuses on automating or creating fixtures for those tasks that are most difficult to perform by hand (for example, the manipulation and precise positioning of the micro parts). The aim is to develop efficient, reliable, yet flexible fabrication techniques. The proposed methods will then constitute part of a minimal set of robust primitives that can be eventually combined to

*This work was funded by NSF DMI-0115091 and NSF IIS-0083472. make a fully automated desktop assembly process for millirobots.

The basic materials chosen to construct these prototype structures are hollow triangular stainless steel beams for the rigid elements and polyester for the flexible elements. During the prototyping stage, where many designs will be tested and discarded, the ready availability and low cost of these materials make them a desirable combination. The rigid elements, made from folding $12.5 \mu \mathrm{m}$-thick stainless steel sheets, are strong yet lightweight and the polyester flexures are able to withstand large deflections and fatigue cycles for a relatively short size. Although a monolithic structure is ideal from the assembly viewpoint, it is difficult to find a single material with desirable mechanical properties for both the rigid and flexible parts. Furthermore, the particular combination chosen here has been successfully used in prototyping millirobots as demonstrated by the Mechanical Flying Insect project [1]. In that case, however, the structures had to be made painstakingly by hand.

The present work extends previous work done at UC Berkeley in the area of microassembly. Shimada et al. [2] describe the development of the Ortho-tweezers used in this work for manipulating tiny blocks and the folding process (done by hand) for the stainless steel beams and provide references to previous work in this area. Thompson and Fearing [3] detail the addition of a user interface and software primitives using force feedback to the Orthotweezers which made the picking and placing of small handling blocks possible automatically.

Figure 1 shows a block diagram of the assembly process from a flat sheet to the final 3-D structure and the current status of automation of each step. The present work describes the efforts made towards the automation of the construction of the triangular beams and the assembly of the beams and flexures into a 2-D assembly. Specifically, in the section on beam construction, we detail the construction of a folding fixture to bend the thin stainless steel sheets and a theoretical analysis done to determine the set of folding angles. In the assembly section, we describe the development of interchangeable pallets where handling blocks 


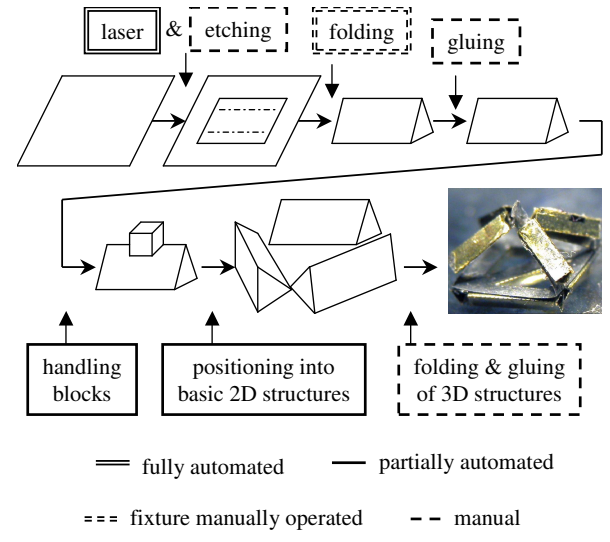

Figure 1: 3-D Structure Assembly Process

are attached to the objects to be manipulated and then the handling block and object combination is transported to its desired position.

The present paper illustrates this procedure through the construction of a prototype for a one DOF wrist for minimally invasive surgery (MIS). These surgical procedures require the development of innovative and dexterous millimeter-scale instruments. Although conventional designs involving miniature machine parts have been proposed (see [4] for example), such mechanisms are difficult to manufacture and assemble and are no longer cost effective beyond a certain level of miniaturization. In addition, rotary and sliding joints used in conventional mechanisms involve surface contact causing unacceptable high friction and wear rates. Compliant mechanisms, in which flexures or flexural hinges replace the conventional revolute joints, are capable of overcoming these disadvantages and are therefore highly suited for this application. For these reasons, we have chosen to demonstrate our current semiautomated rapid prototyping assembly process by building a one DOF wrist for use in MIS instruments. Figure 2 shows a SolidWorks model of the proposed wrist. The present design has been kept simple to illustrate the assembly process, but the same procedure can be applied to more complicated structures as well.

The details of the triangular beam construction and the assembly process as well as the design process used to choose the parameters of the wrist fabricated are given in the sections below.

\section{Triangular Beam Construction}

As mentioned previously, we formed the triangular beams from folding pre-cut and pre-scored $12.5 \mu \mathrm{m}$-thick stain-

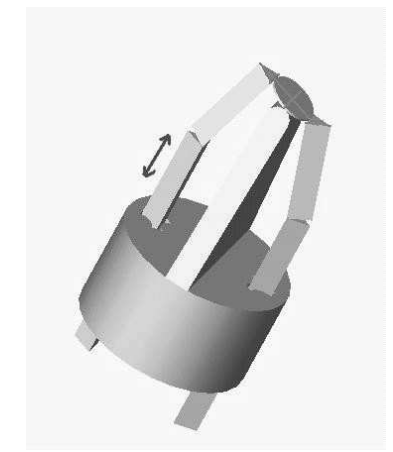

Figure 2: SolidWorks model of the Proposed 1 DOF Wrist

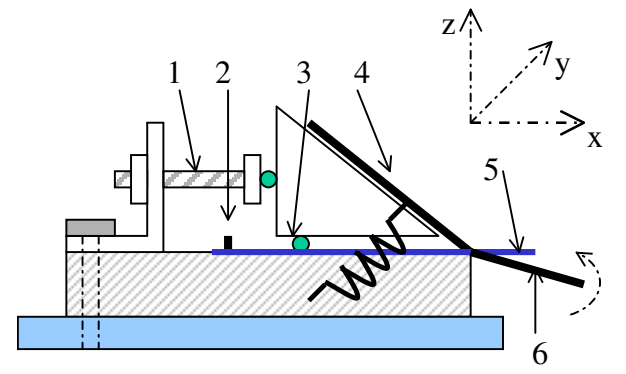

Figure 3: Folding mechanism: (1)clamp-position adjustment screw, (2)alignment pin, (3)clamp point contact, (4)upper razor blade, (5)stainless steel sheet, (6)rotating razor blade

less steel sheets. The folding process, although slower than stamping, provides bending that is more amenable to small, thin sheets. It also allows for greater flexibility in the resulting cross-sectional shape of the beams. The stainless steel sheets were pre-cut and pre-scored using the Microlaze laser micromachining station as described in [2]. The process included pre-coating a small stainless steel piece (approximately $45 \mathrm{~mm} \times 60 \mathrm{~mm}$ ) with polyimide (HD Microsystems, PI2525), blasting the polyimide with the laser, and etching to produce the cut and score lines. The fixture that we developed for producing the folds in the stainless steel sheets is shown in Figure 3 and consists of three basic parts: (1) the mechanism for bending the sheet, (2) the base on which the stainless steel sheet is placed, and (3) the clamp that holds the steel in place and that also serves as an edge against which the sheet is folded.

In order to reduce the springback (for a description of the factors that affect the springback and an approximate way to calculate it, see [2]), an automated folding fixture must provide an almost pure bending moment and minimize the length over which the bending takes place. The folding mechanism pictured in Figure 3 has been designed with 
this aim in mind. A razor blade attached to the clamp applies a line force along the bend line, and a rotating razor blade underneath the steel provides the bending moment. The rotating razor blade is attached to the base structure using simple flexures made of tape. The base provides a hard and flat surface on which to lay the stainless steel piece. The base in the present fixture was molded out of polyurethane with a $200 \mu \mathrm{m}$-thick steel piece glued to the area beneath the clamp. It incorporates two alignment pins to ensure accurate placement of the stainless steel sheet each time a fold is made.

The clamp is perhaps the most critical part of the folding fixture. It must be easy to lift up and place accurately in alignment with the bend line on the stainless steel piece being folded. The clamp's design follows the exact constraint design principles as described in Blanding [5] to avoid over-constraint problems that lead to either loosely fitting or binding parts that are contrary to precise placement. In this case, the clamp uses the exact constraint method of a contact point coupled with a nesting force (provided by tension springs). This method is ideal for parts that are frequently removed and replaced but need to be precisely positioned [5]. In practical use, the clamp is lifted and a pre-cut and pre-scored stainless steel piece is placed on the alignment pins. The clamp is then put down, the springs are put in place to hold it down, and adjustments (using two screws with ball bearings on their tips) are made so that the clamp edge lines up with the steel bend line. Finally, the bottom razor blade is rotated to complete the bend. This process is repeated for all of the bends.

The triangular configuration considered here requires three sequential bends. The folding process in this case consists of bending the sheet metal sequentially at P3, P2, and P1 (Figure 4) until the bent piece just touches the clamp surface at P4. Further rotation of P1P2 by applying an increasing moment causes the structure to flex somewhat and change its angular configuration. Initially, friction prevents the motion of the end P4 along the surface of the clamp, but at some value of the applied moment, the motion would impend. To determine the conditions under which the edge $\mathrm{P} 4$ will always move down (rather than up, as was observed in some initial trials) the clamp surface, we need to carry out a static analysis. It is not sufficient to simply choose the angles so that the sheet is physically angled downward at $\mathrm{P} 4$; whether the impending motion is up or down depends on the direction of the reactive force at P4. All possible resultant reactions must lie within a cone with apex at $\mathrm{P} 4$ and vertical semi-angle $\tan ^{-1} \mu$ (the cone of friction, see Figure 4). For impending motion up or down, the reactive force must lie on the right or left edge of the cone respectively.

We received inspiration from Lu and Akella [6] to model each line shown in Figure 4 as a robotic link. The static

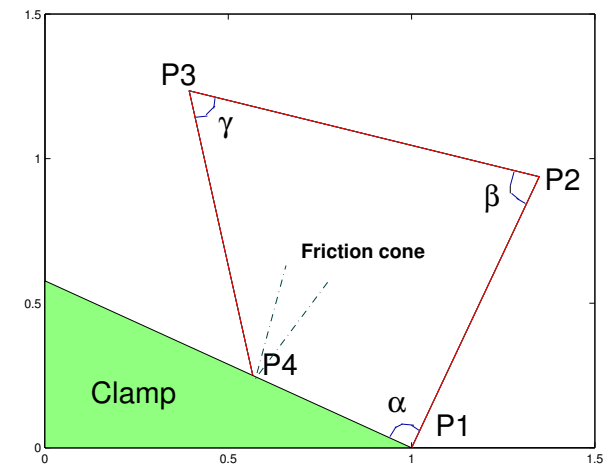

Figure 4: Typical triangular beam folding configuration

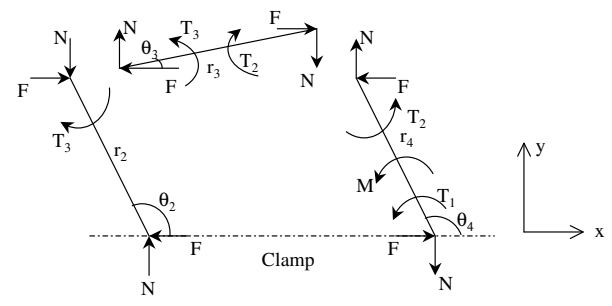

Figure 5: Free body diagram based on a pseudo-rigid compliant mechanism model

analysis presented here is based on the pseudo-rigid compliant mechanism model [7]. In this model, the compliant structure of Figure 4 is replaced by a four bar mechanism with rigid links and torsional springs placed at the joints. In the free-body diagrams of Figure 5 based on this model, the $T \mathrm{~s}$ represent the reactive torques due to the torsional springs. It is easy to verify (by writing the force equilibrium equations) that the magnitudes of the $\mathrm{x}$-components of the reaction forces at each joint are the same; and the same is true for the y-components as well. These components along and perpendicular to the clamp surface are designated as $F$ and $N$ respectively in Figure 5; at $\mathrm{P} 4$ these represent the friction and normal forces. $M$ is the applied moment.

The torques $T_{1}, T_{2}$, and $T_{3}$ depend on the equivalent spring stiffness $k$ and the change in joint angle. The initial values of joint angles $\alpha, \beta$, and $\gamma$ (see Figure 4 ) are determined from the configuration in which $\mathrm{P} 4$ just touches the clamp surface. Representing the initial values by the subscript 0 , the spring torques are given by

$$
T_{1}=k\left(\alpha-\alpha_{0}\right) ; T_{2}=k\left(\beta-\beta_{0}\right) ; T_{3}=k\left(\gamma-\gamma_{0}\right)
$$

At the point of impending motion, the friction force reaches its maximum value $\mu N$ where $\mu$ is the coefficient 
of friction. The experimentally determined value of 0.2 for $\mu$ will be used in the subsequent calculations.

It is convenient to represent the governing equations in a non-dimensional form. The following transformations will be used:

$$
\begin{array}{rc}
M^{*}=\frac{M}{k} & N^{*}=\frac{N l}{k} \\
T_{i}^{*}=\frac{T_{i}}{k} & (i=1,2,3) \\
r_{i}^{*}=\frac{r_{i}}{l} & (i=1,2,3,4)
\end{array}
$$

Here, the starred quantities are non-dimensional and $l$ represents a scaling length. The link lengths $r_{2}, r_{3}$, and $r_{4}$ (see Figure 5) are constant and assumed equal to the scaling length $l$. The variable distance P1P4 is designated as $r_{1}$. The angles $\theta_{i}$ are shown on Figure 5 and are related to the joint angles by simple geometrical relationships. The non-dimensional governing equations are as follows:

$$
\begin{gathered}
\cos \theta_{2}+\cos \theta_{3}-\cos \theta_{4}=r_{1} \\
\sin \theta_{2}+\sin \theta_{3}-\sin \theta_{4}=0 \\
-\left(\gamma-\gamma_{0}\right)-F \sin \theta_{2}-N \cos \theta_{3}=0 \\
\left(\gamma-\gamma_{0}\right)-\left(\beta-\beta_{0}\right)-F \sin \theta_{3}-N \cos \theta_{3}=0 \\
M+\left(\alpha-\alpha_{0}\right)+\left(\beta-\beta_{0}\right)+F \sin \theta_{4}+N \cos \theta_{4}=0
\end{gathered}
$$

The asterisks in the above equations have been dropped for ease of writing, and all quantities are henceforth presumed to be non-dimensional. Equations (3) and (4) arise from geometrical conditions, and Equations (5), (6), and (7) are moment equations written for each of the free body diagrams of Figure 5. This system of nonlinear equations can be solved by a variety of numerical procedures; the method chosen here employs the MATLAB function $f$ solve.

For the solution to have a physical meaning, certain constraints must be satisfied. The normal force should be positive to ensure physical contact at P4. In addition, $\theta_{2}$ must not exceed 180 degrees. Since the direction of the friction force, (see Figure 5) was chosen so as to indicate impending motion down the clamp, a negative value of the friction force would indicate the undesirable situation of impending motion in the upward direction. Out of the feasible set of angle sequences, we use values $\alpha=89, \beta=101$, and $\gamma=60$ degrees in our work. Although currently the folding mechanism is manipulated by hand, the aim is to fully automate it. Thus it is crucial to know a set of angles that work consistently to obtain a triangular cross section (rather than an open sheet) as a finished product.

Once the triangle has been pre-bent into shape, glue is applied with a needle applicator. Then a fixture with $1 \mathrm{~mm}$ equilateral triangle channel cut into it is passed repeatedly over the triangle to hold it in place while the glue dries. The

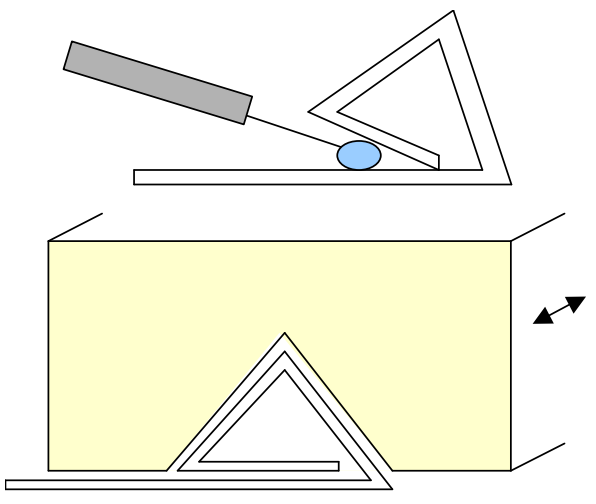

Figure 6: Process used to glue the beams in place

fixture is kept in motion with small back and forth movements to prevent any glue from attaching the triangle to the fixture (See Figure 6).

\section{The 3-D Assembly Process}

Our efforts toward the automatic assembly of these beams into 3-D structures involved the use of the Ortho-tweezers (see Figure 7(a)). Details of this system can be found in [2]. As demonstrated in [2] and [3], the Ortho-tweezers reliably grasp and manipulate blocks. The system's capabilities have been extended to manipulating objects of other shapes and sizes by first attaching a handling block to the object, and then using the Ortho-tweezers to move the block and object combination by grasping and moving the attached block. The handling block is attached to the object using low melting-point wax so that it can be easily removed after the object has been put into position. A successful demonstration of this method for moving strain gages may be found in [3]. The use of handling blocks allows the system to move objects whose high aspect ratio or fragility would preclude them from being handled by conventional tweezers. Thus in our assembly process, the first step is to create a pallet of blocks, wax glue, and the objects that need to be assembled. The Ortho-tweezers are then used to attach the blocks to the objects, thus creating a second interchangeable pallet consisting of the blocks and objects attached together (see Figure 7(b)). In the case where the objects that need to be manipulated are all the same, this step can be done ahead of time and the pallet with block and object combinations can be stored for future use.

For the beam assembly process, the beams are stored in Vgrooves at certain definite angles (e.g. 0, 45, 60, 90, or 120 degrees). These $\mathrm{V}$-grooves are of the same shape as those 


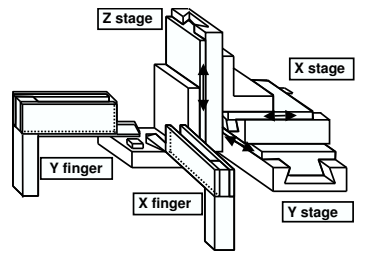

(a)

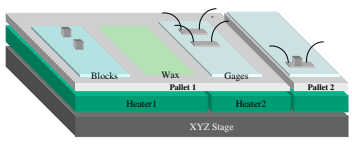

(b)
Figure 7: Ortho-tweezers and sample pallet (a) Orthotweezer configuration $(b)$ sample pallet showing the attachment of handling blocks to strain gages

used in the gluing process during the triangular beam construction. In the fully automated scheme, these same channels can be used. They would simply be flipped over with the triangular beams inside and slid into place in the pallet. A pallet is constructed with the blocks, spin-coated wax (15 $\mu \mathrm{m}$-thick), V-grooves with the triangular beams, and a rubber mold with V-grooves in a 2-D pattern (a flattened version of the desired 3-D structure). We constructed the $\mathrm{V}$-grooves in the 2-D pattern using a 3-D printing machine (3D Systems ThermoJet machine). A semi-automated process then begins where the user identifies the position of the block, wax, beam, and target location. Then an automated process begins where the block is grasped, dipped in wax, and attached to the beam (see Figure 8(a)). Then the block and beam combination is picked up (see Figure $8(b))$ and placed in the appropriate V-groove in the rubber mold of the 2-D pattern (see Figure 8(c)). This step would be repeated as necessary.

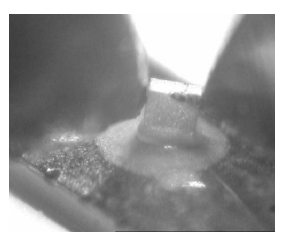

(a)

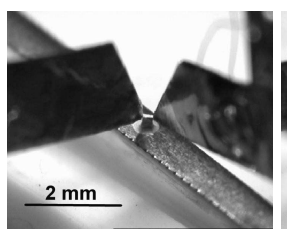

(b)

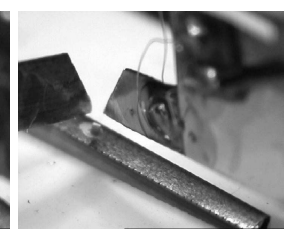

(c)
Figure 8: Beam assembly: (a) handling block attached to the beam $(b)$ block and beam being raised from the Vgroove $(c)$ beam inserted in $\mathrm{V}$-groove of the 2-D pattern

For the construction of the surgical wrist, another pallet was constructed with polyester strips and a 2-D faceplate (laser-cut from $12.5 \mu \mathrm{m}$-thick stainless steel in again a flattened version of the desired 3-D structure) replacing the $\mathrm{V}$-grooves and the 2-D mold. A thin layer of UV-cure glue was placed on top of the 2-D faceplate. Then a similar process as described for manipulating the beams is performed to place the polyester strip $(16 \mathrm{~mm} \times 1 \mathrm{~mm})$ on the 2D faceplate (see Figure 9). Exposure to UV light seals the polyester strips in place. The flexures are created from the polyester strip by the gaps in the stainless steel faceplate below it. Note that in both pallets, the blocks, polyester strips, and the 2-D faceplate are kept on top of Gel-Pak to counteract the sticking force of the parts to the tweezers. (See http://www.gelpak.com for information on Gel-Pak.)

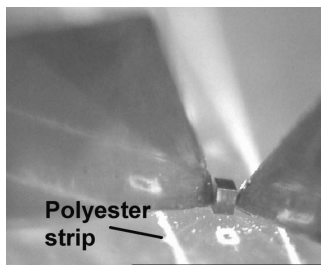

(a)

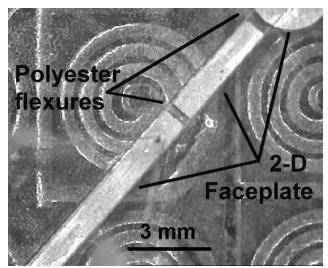

(b)
Figure 9: Attaching the flexures: $(a)$ block and polyester strip being transported $(b)$ strip being put in its place on the 2-D faceplate

Tests of the method described above revealed that it could be successfully performed with the user operating the tweezers through the user interface. However, in the automated procedure, user intervention was sometimes needed at the lifting of the block and object combination and at the final placement of the object. Work is currently being done to make this process more reliably performed without user intervention.

As a final step, the faceplate with the flexure attached is taken and glued on top of the beams in the 2-D mold, removed from the mold, and assembled into the final 3D structure. As the parts being manipulated here are no longer very small, this step is performed by hand. Figure 10 shows the final results. The structure that holds the wrist in place was constructed from polyurethane from a mold fabricated using the ThermoJet machine.

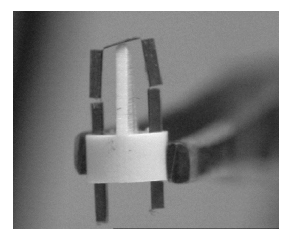

(a)

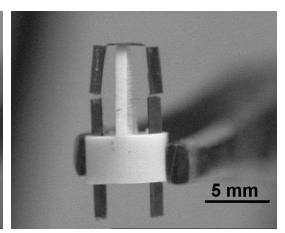

(b)

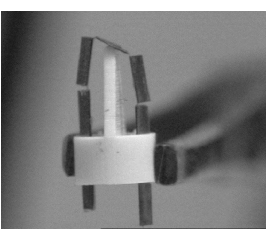

(c)
Figure 10: Wrist prototype shown in different configurations $(a)$ tilted to the left $(b)$ center position $(c)$ tilted to the right

Finally, it should be noted that the above procedure is easily tailored to make other types of 3-D structures. A similar procedure can also be used to attach strain gages to a structure. Further details of the strain gage attachment can be found in [3] and [8]. 


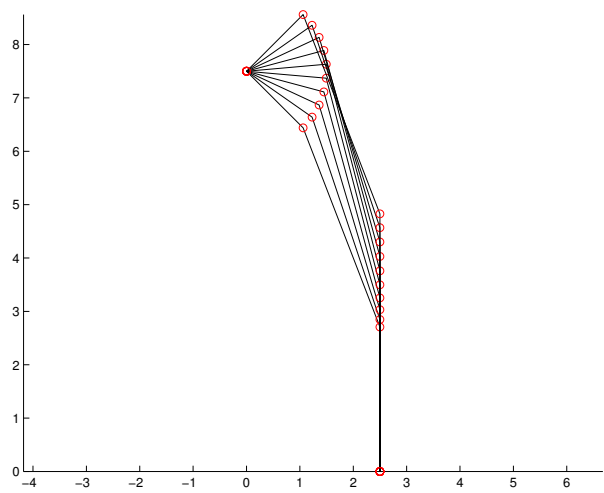

Figure 11: Wrist configurations for platform orientations ranging from -45 to +45 degrees in 10 degree increments

\section{Wrist Design}

This section presents the design process used to determine the parameters for the wrist whose assembly process is described above. Figure 2 shows a schematic of the planar wrist. The two actuator links manipulate the upper platform. The support in the middle provides stability to the platform and takes up some of the load that would otherwise be carried by the two legs. The legs are connected to the upper platform and the actuator links by flexure joints and are both $4 \mathrm{~mm}$ long.

The kinematics and force analysis of the simple mechanism is relatively straightforward. Figure 11 shows the wrist configurations for platform orientations ranging from -45 to +45 degrees. From this geometric study, we determined that the maximum angle that the flexures have to bend through is $90 \mathrm{deg}$. Similar calculations showed that the required stroke length for the actuators is approximately $2.5 \mathrm{~mm}$. Based on an applied torque (as estimated by [9]) of $2.2 \mathrm{~N} * \mathrm{~cm}$, the calculated value of the actuator force is $18 N$.

The stiffness of the flexure may be estimated from elementary beam theory as $E I / d$, where $E$ is the Young's modulus, $I$ is the cross-sectional moment of inertia, and $d$ is the pivot length. The quantities that need to be specified to calculate the stiffnesses are the width $(b)$, the thickness $(h)$, and the length $(d)$. It is note worthy that the above relationship is accurate even when large deflections are involved. For optimum performance, the flexure stiffness should be as small as possible. The stiffness can be reduced by decreasing $E$ and $I$ (since $I$ is proportional to $h^{3}$, an effective way to reduce $I$ is to reduce the flexure thickness) or by increasing the length $d$. The last option is not a desirable one since a long flexure may buckle easily.
For a simple (beam-type) flexure, the appropriate stiffness relations can be found in any strength of materials book and are given by equations (12-16) of Goldfarb and Speich [10]. The quantities that should be considered when designing simple beam flexures include the axial stiffness $(E b h / d)$, the transverse or revolute stiffness $\left(E b h^{3} / 12 d\right)$, and the ratio of axial to transverse stiffness $\left(12 / h^{2}\right)$. Ideally, the last quantity should be as large as possible. The maximum rotation that a conventional flexure can go through before yielding is also an important parameter and is given by $\theta_{\max }=2 d \sigma_{y} / E h$. This last relationship can be easily derived from the formula for maximum bending stresses in a beam subjected to pure bending. Here $\sigma_{y}$ represents the yield stress of the flexure material. As stated earlier, an important additional consideration in flexure design is the buckling of the flexure. Modeling the flexure as a link that is fixed at one end and free on the other, the critical strain as determined from the standard column buckling relationships is given by $\left(\frac{\sigma}{E}\right)_{c r i t}=\frac{\pi^{2}}{48}\left(\frac{h}{d}\right)^{2}$. Also the minimum length of the flexure for a given angle of rotation and thickness is $d_{\min }=\frac{h(\pi-\theta)\left(1-v+v^{2}\right)^{1 / 2}}{2\left(1-v^{2}\right)\left(\sigma_{y} / E\right)}$ (equation 3 from Reference [11]).

It is clear from the above relationships that the material properties play an important part in flexure design. Ideally, the quantity $\sigma_{y} / E$ should be as large as possible. The metals $\left(\sigma_{y} / E=.004\right.$ for stainless steel $)$ are therefore poor candidates for flexures. We choose polyester because of its relatively high $\sigma_{y} / E$ value (.06) and superior adhesion properties. Based on the above calculations, we chose $12 \mu \mathrm{m}$ thick polyester, $1 \mathrm{~mm}$ wide, and $.25 \mathrm{~mm}$ long. Its properties for some of the quantities discussed above is shown in Table 1 . The platform legs can withstand a buckling load of up to $50 N$.

Although a variety of actuation methods are possible, we propose to use SMA actuators because of their small size. The required dimensions of the actuators in the present design can be calculated easily by using the relationships given by Waram [12]. The diameter of the actuator wire is given by $D=(4 F / \pi \sigma)^{1 / 2}$ and the length is equal to $L=$ stroke $/\left(\varepsilon_{1}-\varepsilon\right)$. Here $F$ is the actuator force, $\sigma$ is the design stress, and $\varepsilon_{1}$ and $\varepsilon$ are the high and low strain values. Using a conservative estimate of $25 \mathrm{~N}$ for the actuator force and the stroke length of $4 \mathrm{~mm}(2.5 \mathrm{~mm}$ actual) we need to use actuator wires $400 \mu \mathrm{m}$ in diameter and $80 \mathrm{~mm}$ in length. We used $\sigma=140 \mathrm{MPa}$ (to ensure good cycle life) and $\varepsilon_{1}=0.55 \%$ and $\varepsilon=0.02 \%$. 


\begin{tabular}{|c|c|c|c|}
\hline$k_{\text {axial }} / k_{\text {transverse }}$ & $\theta_{\max }$ & $P_{\text {crit }}$ & $d_{\min }$ \\
\hline $83,333\left(1 / \mathrm{mm}^{2}\right)$ & $143 \mathrm{deg}$ & $6.5 \mathrm{mN}$ & $.30 \mathrm{~mm}$ \\
\hline
\end{tabular}

Table 1: Polyester flexure properties. (The $d_{\text {min }}$ value is conservative since some yielding is permissible.)

\section{Summary and Future Work}

We have developed new methods for the automated assembly of prototype structures, and we describe the construction of a one DOF surgical wrist using this procedure. The wrist mechanism has stainless steel hollow beams for its rigid elements and uses polyester flexures instead of conventional revolute joints. The methods developed for the first step in the structural assembly, the construction of the hollow triangular beams, include the development of a folding fixture to bend stainless steel sheets and the determination of a folding angle sequence. The manipulation of the beams as well as the attachment of the flexures was performed using the Ortho-tweezers system. For this system, pallets were developed with interchangeable parts so that they could be customized to suit each particular assembly. Partial automation of the assembly process is made possible through the use of software primitives. The procedure works well with a user teleoperating the Ortho-tweezers but more work needs to be done to make the procedure completely automated.

Future work will involve building a self-contained desktop rapid prototyping system for millirobots such as the surgical wrist by combining the folding, bonding, and microassembly steps described in this paper. Even though the wrist described in this paper represents a very simple mechanism, the same process can assemble prototypes of more complicated structures. Research could also be done in extending this process in assembling structures of other materials (such as polyurethane and carbon fiber structures).

\section{Acknowledgments}

The authors would like to thank Srinath Avadhanula, Stanley Baek, Domenico Campolo, Steven Jones, Manas Menon, Gabe Moy, Metin Sitti, Erik Steltz, Joe Yan and Robert J. Wood for useful discussions and assistance.

\section{References}

[1] Fearing, R.S., et al., "Wing Transmission for a Micromechanical Flying Insect," IEEE Int Conf on Robotics and Automation, April, 2000.
[2] Shimada, E., et al., "Prototyping Millirobots using Dexterous Microassembly and Folding," Symp on Microrobotics, ASME Int Mech Eng Cong And Exp, Nov. 5-10, 2000, Orlando, FL.

[3] Thompson, J.A., and R.S. Fearing, "Automating Microassembly with Ortho-tweezers and Force Sensing," IROS 2001, Maui, HI, Oct. 29 - Nov. 3, 2001.

[4] Minor, M., and R. Mukherjee, "A Mechanism for Dexterous End-Effector Placement During Minimally Invasive Surgery," ASME Journal of Mechanical Design, vol.141, no.4, pp.472-479, Dec. 1999.

[5] Blanding, Douglass L., Exact Constraint: Machine Design Using Kinematic Principles, ASME Press, New York, 1999.

[6] Lu, Liang and Srinivas Akella, "Folding Cartons with Fixtures: A Motion Planning Approach," IEEE Trans on Robotics and Automation, vol.16, no. 4, pp. 346356, Aug. 2000.

[7] Howell, L. L., and A. Midha, "A Method for the Design of Compliant Mechanisms with Small-Length Flexural Pivots", ASME J of Mech Design, vol. 116, no.1, pp. 280-290, March 1994.

[8] Sahai,R, J. Lee, and R.S. Fearing, "Towards Automatic Assembly of Sub-Centimeter Millirobot Structures," Third International Workshop on Microfactories, Minneapolis, MN, Sept. 16-18, 2002.

[9] Cavusoglu, M.C., et al., "A Laparoscopic Telesurgical Workstation," IEEE Transactions on Robotics and Automation, vol. 15, no. 4, pp. 728-739, Aug. 1999.

[10] Goldfarb, M. and J. E. Speich, "A Well-Behaved Revolute Flexure Joint for Compliant Mechanism Design," Journal of Mechanical Design, vol. 121, pp. 424-429, Sept. 1999.

[11] Yan, J., et al., "Towards Flapping Wing Control for a Micromechanical Flying Insect," IEEE Int Conf on Robotics and Automation, pp. 3901-3908, Seoul Korea, May 21-26, 2001.

[12] Waram, T. C., "Actuator Design Using Shape Memory Alloys" ISBN 0-9699428-0-X (Available through Mondo-tronics, 524 San Anselmo Ave., \#107, San Anselmo, CA 94960, (415) 455-9330, FAX (415) 4559333). 